\title{
Turning the Tables on Partial Credit: Computer Aided Exam with Student Reflection for Partial Credit (CAESR4PC)
}

\section{Dr. Jeffrey Lloyd Hieb, University of Louisville}

Jeffrey L. Hieb is an Associate Professor in the Department of Engineering Fundamentals at the University of Louisville. He graduated from Furman University in 1992 with degrees in Computer Science and Philosophy. After 10 years working in industry, he returned to school, completing his Ph.D. in Computer Science Engineering at the University of Louisville's Speed School of Engineering in 2008. Since completing his degree, he has been teaching engineering mathematics courses and continuing his dissertation research in cyber security for industrial control systems. In his teaching, Dr. Hieb focuses on innovative and effective use of tablets, digital ink, and other technology and is currently investigating the use of the flipped classroom model and collaborative learning. His research in cyber security for industrial control systems is focused on high assurance field devices using microkernel architectures.

\section{Dr. Campbell R Bego, University of Louisville}

An instructor and postdoctoral researcher in engineering education, Campbell R. Bego, $\mathrm{PhD}, \mathrm{PE}$, is interested in improving STEM student learning and gaining understanding of STEM-specific learning mechanisms through controlled implementations of evidence-based practices in the classroom. Dr. Bego has an undergraduate Mechanical Engineering degree from Columbia University, a Professional Engineering license in the state of NY, and a doctorate in Cognitive Science. 


\title{
Turning the Tables on Partial Credit: Computer Aided Exam with Student Reflection for Partial Credit (CAESR4PC)
}

\begin{abstract}
This full-length research paper describes a new type of exam, the Computer Assisted Exam with Student Reflection for Partial Credit (CAESR4PC). CAESR4PC combines the automatic grading of computer-based exams with a partial credit mechanism that rewards students for post-exam reflection. Intended to be a replacement for hand graded traditional essay style exams, the grading effort once applied to assigning partial credit and giving formative feedback is now spent reviewing student post-exam reflections. Descriptive statistics and quasi-experimental study results from an initial implementation of this pedagogical technique in a linear algebra course for engineering students are presented. While the CAESR4PC approach is developed with math courses in mind, it is equally applicable to other subjects that have a significant amount of mathematical/analytical content.
\end{abstract}

\section{Introduction}

There are probably as many different types of math exams as there are math instructors. Across the variety of types in undergraduate mathematics, exams are primarily seen by students and faculty as summative assessments. Dixson and Worrell [1] describe summative assessments as cumulative exams that "capture what a student has learned" (p. 156). Formative assessments, by comparison, "provide feedback to teachers and students to help learn more effectively" [1], page 154). When students are expected to both perform and learn from their mistakes on exams, those exams are both summative and formative in nature. However, when student grades depend heavily on exam averages, which is typically the case in undergraduate math courses, students are most likely to view exams as summative assessments unless otherwise directed.

Despite instructors' aspirations, students who have mastered the procedural tasks for a given exam - and even those who have gained a deep understanding of the relevant concepts - still make errors when working out answers to exam questions. Different types of exams handle these mistakes differently. For the purpose of this paper, common math exam types are categorized into three groups: 1) essay, 2) multiple choice, and 3) computer assisted. These types vary primarily along parameters of a) scoring entity and b) partial credit. Exam type is often selected along these parameters for practical reasons such as class size and grading time required (see Cherkas and Roitberg [2]).

The possibility for exams to be used as formative assessments exists. One well-known strategy for directing students towards a formative use of exams is using Exam Wrappers [3] which engage students in self-reflecting on their work. In addition to the three primary types of exams, exam wrappers are described in detail below. 


\section{Essay exams}

Traditional essay exams in math are scored by instructors or graders and characterized by a "show-your-work" grading system. This type of exam will appear on paper as a math question or word problem followed by substantial space for student work. Both the student work and the final answer are scored or graded, and often the procedural steps may be worth more than the final answer. A typical example of an essay type question, student response, and partial credit grading are shown in figure 1.

$$
\begin{aligned}
& \text { \#8 [17 pts.] Given the function, } f=g(t, u, v, w)=u^{2} w+t^{2} u v^{3}+t v^{2} w \text { subject to the constraint } \\
& \frac{w^{2}}{t^{3}}+t^{3} u^{2} w=5 \text { find the following partial derivatives or explain why they do not exist. } \\
& \left(\frac{\partial f}{\partial w}\right)_{t, v}=\frac{\partial f}{\partial t}\left(\frac{\partial t}{\partial w}\right)_{t, v}^{0}+\frac{\partial f}{\partial u}\left(\frac{\partial u}{\partial w}\right)_{t, v}+\frac{\partial f}{\partial v}\left(\frac{\partial v}{\partial w}\right)_{t, v}^{c}+\frac{\partial f}{\partial w}\left(\frac{\partial w}{\partial w}\right)_{t, v}=1 \\
& =0+\left(2 u v+t^{2} v^{3}\right)\left(2 t^{3}+a w\right)+0+\left(u^{2}+t v^{2}\right)(1) \\
& =\left(2 u w+t^{2} v^{3}\right)\left(2 t^{3} u w\right)+\left(u^{2}+t v^{2}\right)
\end{aligned}
$$

Figure 1. An essay exam question with student response (from Calculus III).

The figure shows that the instructor has identified an error, although some of the student work is correct. The instructor is left to determine the amount of partial credit given on any problem with a mistake or error. In some cases, determining partial credit is straight forward. In others the need to pay attention to relative partial credit assigned to different mistakes, makes it much more difficult. Difficulty is also introduced when there are multiple graders for a given exam. Rubrics and scaffolded problems (with identified intermediate answers) can help to improve the meaningfulness and consistency of partial credit, however, the challenges of essay-style exams are experienced by faculty on regular basis.

Essay style exams do provide an opportunity for student learning. When exams are returned, the visible marks on the student work can provide formative feedback to students about their understanding. In some cases, instructor solutions are provided as well, which provides another opportunity for formative feedback. Instructors can tell students to use the feedback formatively, to help their learning, but that does not mean students do. It is safe to assume that, left to their own devices, most students (particularly those who are not self-regulated learners) view exams only as summative, even when they ask for one of their answers to be regraded.

\section{Multiple choice exams}

There are many variations on multiple choice tests (MCT), however the primary grading mechanism is the same: students get credit if they select the correct answer from a list of choices. MCTs are attractive because they can be scored automatically. Scantron ${ }^{\circledR}$ is a common method, 
and more recently, most learning management systems provide automatic scoring of online multiple-choice questions. Usually MCTs do not offer partial credit, since student work is not reviewed. Figure 2 captures the essential characteristics of the MCT question in post-secondary instruction.

$$
\begin{aligned}
& \text { \#8 Find }\left(\frac{\partial f}{\partial w}\right)_{t, v} \text { at the point }(t, u, v, w)=(1,1,1,1) \text { if the function } \\
& f=g(t, u, v, w)=u^{2} w+t^{2} u v^{3}+t v^{2} w \text { subject to the constraint } \frac{w^{2}}{t^{3}}+t^{3} u^{2} w=5 \\
& \begin{array}{llll}
\text { a) } \frac{5}{2} & \text { b) }-\frac{5}{2} & \text { c) } 8 & \text { d) } 13
\end{array}
\end{aligned}
$$

Figure 2. An MCT question.

MCTs do not typically include partial credit. Cherkas and Roitberg [2] did develop a partial credit multiple choice test (PCMCT) where partial credit is given for the selection of a specific distractor among the possible responses (an incorrect answer representing a common mistake), however this method is not widely used. Even without partial credit, and without instructors identifying specific mistakes within student work, the results of multiple-choice tests can be used formatively, particularly if students have kept their work. However, it also safe to assume most students view multiple choice exams only as summative.

\section{Computer Aided Exams (or online testing)}

The maturity and availability of computer algebra systems has allowed a new category of exams to emerge [4]: the computer aided exam (CAE), also known as the online exam. In CAE exams, students can enter an expression into an online form to respond to a traditional essay style question. The computer system grades the response, recognizing equivalent expressions, possibly giving partial credit for un-simplified expressions. Like MCTs, answers are typically scored right or wrong. Figure 3 shows a typical CAE question and an answer entered by the student. In this example, the answer is scored incorrect, but the student has made a minor mistake, pairing the wrong eigenvector with each eigenvalue.

In some cases, CAEs can award partial credit for non-simplified answers, and partial credit is possible for a single question with multiple parts (similarly to multiple choice exams). With respect to partial credit, CAEs do not differ significantly from multiple choice exams; students receive full credit for a correct answer, and no points for an incorrect answer. Having students answer intermediate questions (scaffolding the problem) can be used in both CAEs and MCTs to allow a form of partial credit. There are no differences between MCTs and CAEs when it comes to formative feedback, because neither has formative feedback built in, though it is possible for students to use their scores to determine where to focus their study. The main advantage of CAEs is that students cannot work backward from the answer or use heuristics to eliminate wrong answers. Students must work the problem to find an answer. 
Solve the initial value problem $\mathbf{x}^{\prime}(t)=A \mathbf{x}(t)$ for $t \geq 0$, with $\mathbf{x}(0)=(4,8)$.

$$
A=\left[\begin{array}{ll}
2 & 3 \\
1 & 4
\end{array}\right]
$$

Solve the initial value problem.

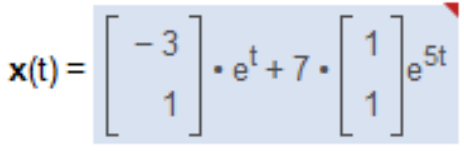

Figure 3. A CAE question.

\section{Post-Exam Reflection: Exam Wrappers}

First introduced by Marsha Lovett [3], exam wrappers have been used and studied in many different disciplines, including math and computer science [5-8]. Exam wrappers are a metacognitive, formative assessment practice where students complete a structured reflection after their graded exams are returned. Designed to promote self-reflection and improve studyhabits, exam wrappers are typically holistic, asking students to provide a short reflect along one or more of the three themes identified by Lovett: reflecting on preparation, kinds of errors, and changes for next time. Exam wrappers are purely formative in nature, although in some courses completion of exam wrappers is a small part of the course grade [5] and in others' students can earn extra credit [7]. The efficacy of exam wrappers is mixed [5-8]; study-strategies identified by students in their exam wrappers are not always adopted, positive results were sometimes selfreported, and in one quasi-experimental study [8] comparison of performance on the final exam showed no statistical difference. However, as Stephenson, et al. [8] point out, the lack of significant results should not preclude the use of exam wrappers, rather expectations about their benefits should be approached with caution and other related approaches should be explored.

Nieminen, et al. [9] introduce a more radical use of reflection. They developed a Blended Reflective-Centered Learning Environment based exclusivity on reflection. Rather than traditional exams, the students in their Linear Algebra class completed a variety of formative, digital self-assessments. They were motivated by research about creating learning environments (classes) that cultivate deep learning. Their analysis found that deep learning was reported (by students) to be higher after the course and that the main contextual factor associated with enhancing deep learning was their innovative assessment.

\section{The Computer Assisted Exam with Student Reflection for Partial Credit (CAESR4PC)}

In CAESR4PC, students first take a computer assisted exam with blank paper to work out their answer in an essay style format. CAESR4PC exam questions can be delivered through any online assessment platform such as WebCT®, WileyPlus ${ }^{\circledR}$, MapleTA ${ }^{\circledR}$, or MyMathLab ${ }^{\circledR}$. Exams are initially graded by the computer system based on binary (right/wrong) answers. 
Following the exam, student work is digitized (scanned) to an online system that allows student to view (but not alter) their work. Students are given access to their work digitally at the same time they are given access to exam results (online). At that time, they can review their work, focusing on the answers that were incorrect. Students are then encouraged to complete a formative exam wrapper task for additional partial credit. The exam wrapper task includes identifying and categorizing mistakes, and outlining how to do the problem correctly. Students must identify the type of error they made and provide some discussion that demonstrates they understand their error and what is required to correctly find the solution or how the error affected their answer. Students can choose to do this for each problem, though they are not required to do so, with partial credit being given on a per question basis. This unique combination of computer aided exam and exam wrapper reflection task is expected to promote deep learning and improve study habits. The workflow of CAESR4PC is shown in figure 4 below.

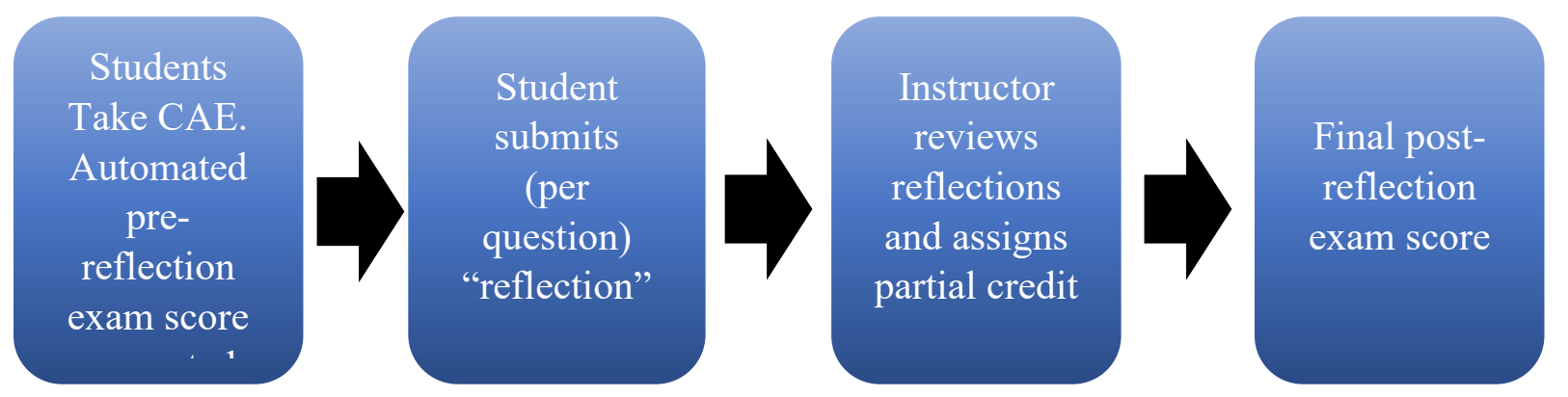

Figure 4. Workflow for CAESR4PC.

Inspiration for CAESR4PC began with the recognition that there is a genuine need for partial credit, because students make mistakes on exams, yet when instructors (or graders) assign partial credit, the opportunity for students to learn from the mistake is likely under-emphasized. If instead, the student had to identify their own errors to earn partial credit, they might be more likely to learn from their mistake. Furthermore, student work is frequently difficult to follow or understand. It is possible that in some cases, poor communication of work might benefit the student when instructors are assigning partial credit. The opposite is the case when students must read and understand their own work: clear documentation of work is rewarded. This inversion should motivate students to make the solutions they submit easier to read and understand.

In CAESR4PC, the amount of partial credit varies by type of error. For example, $95 \%$ credit can be obtained if the student made only minor or careless errors, whereas another amount (e.g., $40 \%$ ) can be obtained for a more fundamental type of error. It is recommended that there only be two levels for partial credit so that once students differentiate between a minor or a fundamental error, they are not motivated to select a specific type of fundamental error because its partial credit percentage is higher. Rather than have instructors assign partial credit individually to each student for each problem, CAESR4PC places that burden back on students, rewarding them with a specific partial credit amount (consistent for all students in the course) for participating in the formative assessment strategy of categorizing and explaining their mistakes. Types of errors can vary by instructor and course but must at least differentiate between the two broad types of errors: minor or careless errors versus substantial misunderstanding. 
CAESR4PC capitalizes on the benefits of computer aided exams while enabling a formative assessment structure. The traditional work of grading essay style exams is replaced with processing post-exam reflections (which were called regrade requests in the implementation). Grading is less difficult for instructors because they need only to agree with students about the type of error that was made, which determines the amount of partial credit.

\section{A quasi-experimental study of CAESR4PC in linear algebra for engineering}

In the summer of 2019, CAESR4PC was implemented for the first time in Linear Algebra for Engineering, a two-credit hour course for engineering students taught at a large research Institution in the Midwest. To determine the impact of this innovative pedagogical practice, student performance was compared to the prior year (2018) in which essay-style exams were used. The same instructor taught the course in both years, used the same book, and implemented the same flipped course structure. The remainder of this paper is dedicated to describing the methodological details and results from this initial implementation study.

\section{Participants}

All students who were enrolled in Linear Algebra for Engineering in either summer 2018 or summer 2019, took the final exam, and for which an ACT Math score was available were participants in this study 2018( $N=96)$ and $2019(N=91)$. Engineering students at this institution attend class year-round and summer courses are a normal part of experience as students. These student groups did not differ in mean ACT math score $F(185,1)=0.134, p=0.714$, partial eta squared $=0.001$.

\section{Materials}

Course structure and content. Linear Algebra for Engineers was taught in both years using a flipped classroom structure. Students completed assignments before class, usually a combination of videos, reading, and practice problems. These pre-class assignments were all assigned using the online system MyMathLab®, which is available for the course text: Linear Algebra and its applications [10]. During class meetings, students work in teams first using a classroom response system (Learning Catalytics) and then later as smaller teams (pods) on a related class activity problem.

Exams in 2018. There were three 75 minute exams in 2018. Each exam was an essay style exam where students were prompted to provide answers and solutions. Each exam was cumulative. Exams were graded by the instructor with help from student graders. Students were required to show work for full credit. On each exam the instructor or student grader assigned partial credit using the instructor solutions, which were marked up with suggested point distributions, as guide.

Exams in 2019. There were four 75 minute exams in 2019, and each exam followed the CAESR4PC format. An explanation and discussion of the CAESR4PC format for semester exams was provided in the syllabus and discussed the first day of class and the class meeting before the first exam. The post-exam reflection was implemented as a regrade request. The regrade request explanation in Appendix $\mathrm{A}$ is identical to the information provided in the 
syllabus and with each the exam. For each of the four semester exams, students were given paper to work out the questions in the MyMathLab Exam, with space for each question clearly indicated. The work was collected and scanned into PDF format. The PDFs were then loaded into GradescopeC (https://www.gradescope.com/) and made available for students to review. Students were reminded that they could request regrades for questions that were scored wrong, and instructed to use Gradescope's request regrade feature.

(https://www.gradescope.com/help\#help-center-item-student-regrades).

Final Exam. The final exam from the summer of 2018 was used again in the summer of 2019, with only minor modifications to a few questions. The summer 2018 final exam was graded in a more tradition fashion (no rubrics, but scored against a worked solution). The final exam in 2019 was scored using rubrics developed specifically for each question, to keep partial credit consistent with the grading of the exams during the semester. For all years, final exams are not returned to students.

Regrade requests (exam wrapper post-exam reflection). Students were given four different types of errors to choose from: 1) careless or minor error, 2) incomplete procedure or solution, 3) significant computational error, 4) fundamental misunderstanding. Information about these categories and the amount of partial credit associated with each was provided in the syllabus and with each exam, that information is replicated in Appendix A. Careless or minor errors received 95\% partial credit, all other types of errors received the same amount of partial credit (fading from $40 \%$ on the first exam to $20 \%$ on the last exam). The assigning of $95 \%$ partial credit for minor or careless errors recognizes that student and instructors alike make minor or careless errors, and such errors should not have a significant penalty on in a summative assessment. The three different types of fundamental mistakes emerged from the instructor's experience grading Linear Algebra exams as well as exams in other engineering mathematics courses.

Differentiating between the three was intended to help students with the metacognitive task of reflecting on their mistakes, rather than focusing on the correct solution. Keeping the partial credit consist among all categories other than minor or careless error was designed to discourage students from seeking to earn more partial credit by strategically characterizing their errors. Students used the request regrade button in Gradescope $(\mathcal{C}$ to initiate the regrade process. Figure 5 shows screen clips and images from all parts of the CAESR4PC workflow.

\section{Results}

\section{Descriptive Results for CAESR4PC}

Table 1 shows the average for each exam before and after regrades were processed. Table 2 shows the number of regrade requests for each exam, the total points returned (partial credit) for each exam and the average points returned per request. 


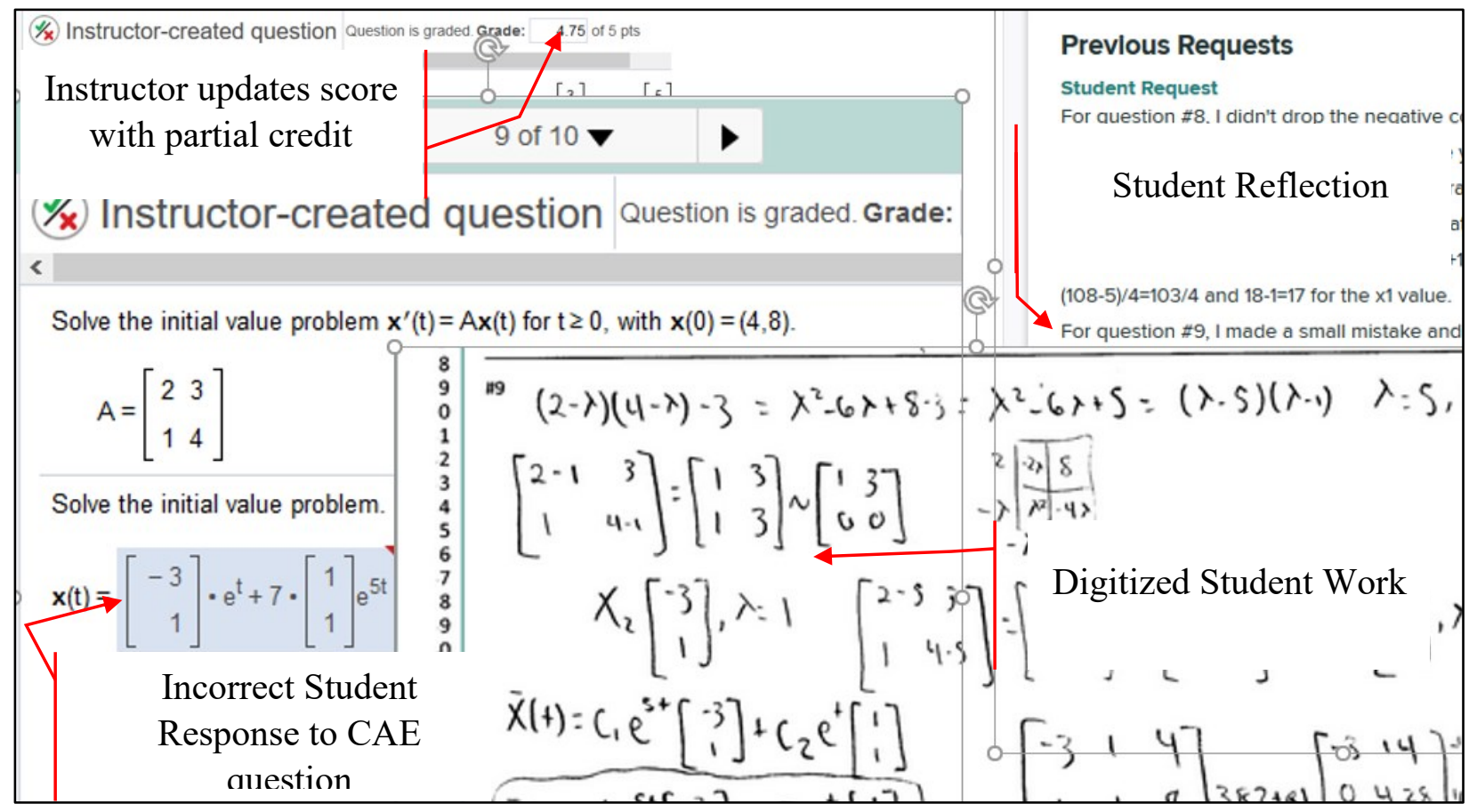

Figure 5. Exam question in MyMathLab, student work on that problem and the students regrade request in Gradescope.

Table 1. Exam averages (in percent), for 2019 includes score before and after regrading.

\begin{tabular}{|l|l|l|c|}
\hline \multicolumn{2}{|c|}{2018} & \multicolumn{2}{c|}{$\mathbf{2 0 1 9}$} \\
\hline \multirow{2}{*}{ Exam 1 } & \multirow{2}{*}{83.20} & Exam 1 Before Regrades & 94.93 \\
\cline { 3 - 4 } & \multirow{2}{*}{ Exam 2 } & Exam 1 After Regrades & 95.51 \\
\hline \multirow{2}{*}{ Exam 3 } & & Exam 2 Before Regrades & 79.31 \\
\cline { 3 - 4 } & \multirow{2}{*}{78.30} & Exam 2 After Regrades & 83.47 \\
\cline { 3 - 4 } & & Exam 3 Before Regrades & 73.24 \\
\cline { 3 - 4 } & & Exam 3 After Regrades & 85.61 \\
\cline { 3 - 4 } & & Exam 4 Before Regrades & 67.12 \\
\cline { 3 - 4 } & Exam 4 After Regrades & 80.73 \\
\hline
\end{tabular}

Table 2. Regrade request descriptive statistics.

\begin{tabular}{|l|l|l|l|l|}
\hline & \multicolumn{1}{|c|}{ Exam 1 } & \multicolumn{1}{|c|}{ Exam 2 } & \multicolumn{1}{|c|}{ Exam 3 } & \multicolumn{1}{c|}{ Exam 4 } \\
\hline Number of regrade requests & 17 & 81 & 141 & 147 \\
\hline $\begin{array}{l}\text { Total points returned (partial } \\
\text { credit) }\end{array}$ & 52.29 & 379.20 & 1097.16 & 1197.80 \\
\hline $\begin{array}{l}\text { Average points returned per } \\
\text { request }\end{array}$ & 3.08 & 4.68 & 7.78 & 8.15 \\
\hline
\end{tabular}




\section{Comparison between Years}

Performance on the final exam was analyzed using a one-way ANOVA with a between subjects factor of year $(2018,2019)$, which varied by exam type (essay style, CAESR4PC). The difference between the mean final exam performance in 2018 and 2019 approached significance, $F(185,1)=3.48, p=.064$, partial eta squared $=0.018$. An ANCOVA was also performed with ACT score as a covariate and results were similar. Means and confidence intervals are shown in figure 6 below.

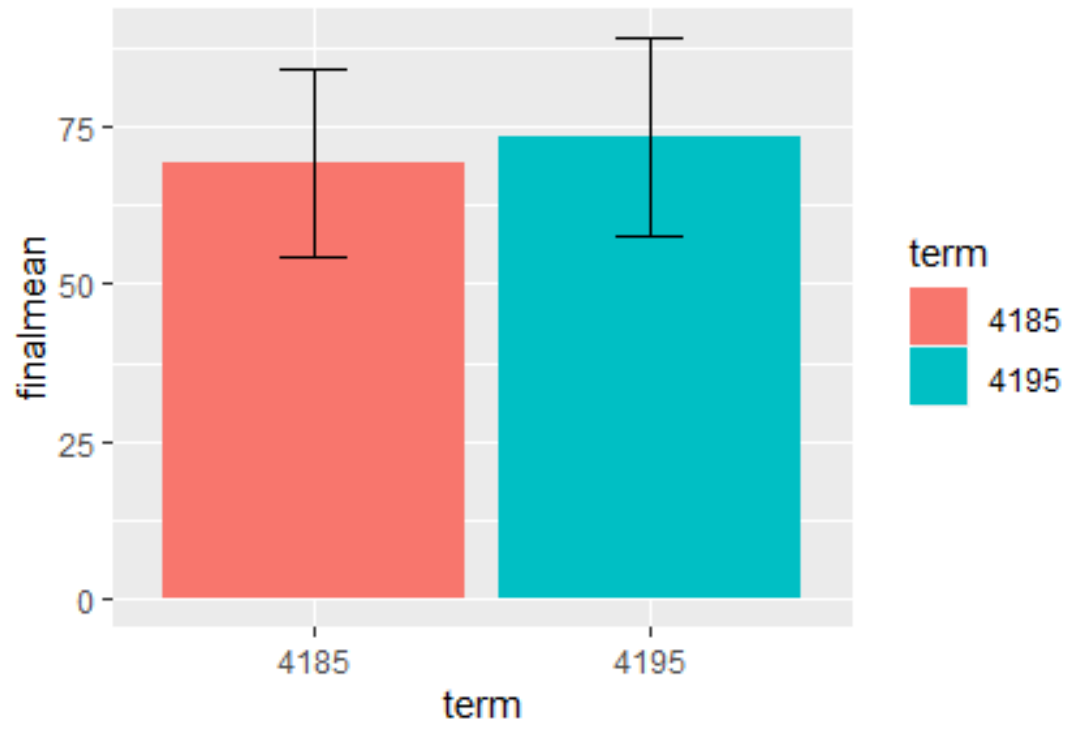

Figure 6. Average final exam performance for years 2018 and 2019, which varied by exam type of semester exam. Error bars represent confidence intervals.

\section{Discussion and Future work}

The study results indicate that the CAESR4PC exam type was beneficial for student learning in the initial implementation in Linear Algebra for Engineers. Improvements in the implementation are likely to increase the effectiveness of this method, perhaps resulting in a statistically significant difference.

The number of regrade requests ( 452 overall) demonstrated that students bought in to the partial credit mechanism. Students did not request many regrades on Exam 1, likely because the average was so high (94.5\%). As performance decreased on the CAE exams over the course of the semester, the regrade requests increased. In addition to this trend of increasing the number of regrade requests, the average points returned per regrade request increased as well. There are several possible interpretations of this increase. Since exam questions are not equally weighted (some questions are worth more points), and there are typically few questions on the later exams, this could be only a product of the point distribution. It might also indicate that the frequency of careless mistakes trended up while the frequency of fundamental mistakes went down. It is probably a combination of both, and additional investigation is needed to further understand the increase in the number of regrade requests and the increase in partial credit. 
The instructor, who reviewed all regrade requests, was surprised that more often than expected, students made minor errors but categorized them as fundamental mistakes. To better understand how CAESR4PC impacted students in this class, future work will examine individual students results in more detail. This study did not look at the quality of student work over time (along both readability and clarity) but when students must identify their mistakes by reflecting on their own work, it seems reasonable to assume they could be motivated to make their work neater and clearer. Future work will analyze of the quality of work done on the exams in the pilot study and explore making that a more explicit part of the reflection. In addition, it is worth mentioning that one student commented that this type of exam reduced his/her test anxiety.

This study is limited to a single implementation at a single university, but the promising results are encouraging for adoption perhaps across many undergraduate STEM courses.

\section{Conclusions}

The CAESR4PC exam type is a promising pedagogical technique that has its origins in exam wrappers. The technique could benefit student learning and possibly reduce instructor grading time and increase grading consistency. The workflow that includes a CAE, student reflection, and instructor regrades takes advantage of modern educational technology as well as evidencebased learning strategies. An initial implementation of CAESR4PC in Linear Algebra for Engineers successfully increased the final exam score. It is possible that the CAESR4PC had other effects as well, such as improving the quality of student work, increasing conceptual understanding, and decreasing student anxiety. This method is well worth exploring in more detail in mathematics and other courses.

\section{References}

[1] D. D. Dixson and F. C. Worrell, "Formative and summative assessment in the classroom," Theory into practice, vol. 55, no. 2, pp. 153-159, 2016.

[2] B. M. Cherkas and J. Roitberg, "Humanizing the multiple-choice test with partial credit," International Journal of Mathematical Education in Science and Technology, vol. 24, no. 6, pp. 799-812, 1993.

[3] M. C. Lovett, "Make exams worth more than the grade," Using reflection and metacognition to improve student learning: Across the disciplines, across the academy, pp. 18-52, 2013.

[4] T. Passmore, L. Brookshaw, and H. Butler, "A flexible, extensible online testing system for mathematics," Australasian Journal of Educational Technology, vol. 27, no. 6, 2011.

[5] M. Craig, D. Horton, D. Zingaro, and D. Heap, "Introducing and evaluating exam wrappers in CS2," in Proceedings of the 47th ACM Technical Symposium on Computing Science Education, 2016, pp. 285-290.

[6] K. J. Chew, H. Chen, B. Rieken, A. Turpin, and S. Sheppard, "Improving students' learning in statics skills: Using homework and exam wrappers to strengthen self-regulated learning," in ASEE Conferences. doi, 2016, vol. 10, p. 25633. 
[7] P. G. Gezer-Templeton, E. J. Mayhew, D. S. Korte, and S. J. Schmidt, "Use of exam wrappers to enhance students' metacognitive skills in a large introductory food science and human nutrition course," Journal of Food Science Education, vol. 16, no. 1, pp. 28-36, 2017.

[8] B. Stephenson, M. Craig, D. Zingaro, D. Horton, D. Heap, and E. Huynh, "Exam wrappers: Not a silver bullet," in Proceedings of the 2017 ACM SIGCSE Technical Symposium on Computer Science Education, 2017, pp. 573-578.

[9] J. H. Nieminen, J. Häsä, J. Rämö, and L. Tuohilampi, "Replacing exam with self-assessment: Reflection-centred learning environment as a tool to promote deep learning," in Proceedings of the 21st Annual Conference on Research in Undergraduate Mathematics Education. San Diego: RUME, 2018.

[10] D. C. Lay, "Linear Algebra and its applications 5th edition," ed: Pearson, 2016. 


\section{Appendix A: Request for regrade MyMathLab question.}

For any question in MyMathLab that you get scored completely or partially incorrect, you can review your work in gradescope and make a regrade request for that question in gradescope, one regrade request per question. Regrade requests must identify the type of error, the location of the error when relevant, discuss the error made, and provide an explanation of what is required for a correct and complete solution. "I made a mistake" is not an explanation. You instructor will need to agree with you about the type of error if points are to be returned.

\begin{tabular}{|l|l|}
\hline \multicolumn{1}{|c|}{ Type of Error } & \multicolumn{1}{c|}{ Characteristics } \\
\hline $\begin{array}{l}\text { Careless or minor } \\
\text { error(s) }\end{array}$ & $\begin{array}{l}\text { The work shows a clear understanding of the problem and mathematical } \\
\text { components present in this problem, however the correct answer was not } \\
\text { found due to minor errors, often in arithmetic. } \\
\text { Examples: mistakes in multiplication or division, copying down part of the } \\
\text { problem incorrectly or transcribing numbers or expressions. }\end{array}$ \\
\hline $\begin{array}{l}\text { Incomplete procedure } \\
\text { or solution }\end{array}$ & $\begin{array}{l}\text { There is some work that is correct and contributes to addressing the } \\
\text { question posed beyond copying down the questions. } \\
\text { An important component of the complete solution is missing. } \\
\text { Example: In an optimization problem finding the interior critical points but } \\
\text { not the boundary points. }\end{array}$ \\
\hline $\begin{array}{l}\text { Significant } \\
\text { Computational Error }\end{array}$ & $\begin{array}{l}\text { The works shows understanding of the concepts needed to solve the } \\
\text { problem but the computation of a final or intermediate result indicates a } \\
\text { fundamental lack of understanding about that computation, especially if it } \\
\text { is a computation presented in this class. } \\
\text { Example: skipping the inner term when finding a derivative using the }\end{array}$ \\
\hline $\begin{array}{l}\text { Fundamental } \\
\text { misunderstanding }\end{array}$ & $\begin{array}{l}\text { The answer has little or no relation to the question or inappropriate } \\
\text { concepts are used and possibly implemented incorrectly. } \\
\text { Example: The question is a related rate problem and the work is for a } \\
\text { relative error problem or an optimization problem. }\end{array}$ \\
\hline
\end{tabular}

The percentage of points returned for different types of errors.

\begin{tabular}{|l|c|c|c|c|}
\hline Type of Error & \% returned Exam 1 & \% returned Exam 2 & \% returned Exam 3 & \% returned Exam 4 \\
\hline $\begin{array}{l}\text { Careless or minor } \\
\text { error }\end{array}$ & $95 \%$ & $95 \%$ & $95 \%$ & $95 \%$ \\
\hline $\begin{array}{l}\text { Incomplete } \\
\text { procedure or } \\
\text { solution }\end{array}$ & $40 \%$ & $40 \%$ & $30 \%$ & $20 \%$ \\
\hline $\begin{array}{l}\text { Significant } \\
\text { Computational } \\
\text { Error }\end{array}$ & $40 \%$ & $40 \%$ & $30 \%$ & $20 \%$ \\
\hline $\begin{array}{l}\text { Fundamental } \\
\text { misunderstanding }\end{array}$ & $40 \%$ & $40 \%$ & $30 \%$ & $20 \%$ \\
\hline
\end{tabular}

